\title{
On MEASUREMENTS OF Kinetic PARAMETERS IN A MiCROFLUIDIC REACTOR
}

\author{
Matthew B. Kerby ${ }^{2}$, Robert S. Legge ${ }^{1}$, Anubhav Tripathi ${ }^{1}$ \\ 1 Chemical and Biochemical Engineering, Brown University, Providence, RI 02912 \\ 2 Biomedical Engineering, Brown University, Providence, RI 02912
}

\section{$\underline{\text { Supplementary Material }}$}

\section{Chip fabrication and design:}

A 4-channel double mask shown in figure 1 was drawn using AutoCAD 2004 (Autodesk) and printed (CadArt Services, Poway, CA) on a transparency at 20kdpi. Table 1 lists the dimensions of each channel shown in the mask design in figure 1. The microfluidic pattern was transferred into Schott Borofloat 33 borosilicate glass using standard photolithography and fluoride wet etch chemistries as described elsewhere [1]. An unetched glass piece $(50 \mathrm{~mm}$ square) with a pattern of drilled holes (SI Howard Glass, Worcester, MA), which created reagent ports, was thermally bonded to the channel plate creating a twolayer closed-channel borosilicate chip. A Teflon caddy holds the glass chip against a metal backing plate, which applies uniform pressure to o-rings and seals the caddy and top glass plate. The pressure over the microfluidic chip reservoirs is controlled by an 8-port cartridge connected to corresponding pressure lines from the control system.

Table 1: Geometric configuration of the chip

\begin{tabular}{llll}
\hline Channel & Length $(\mu \mathrm{m})$ & $\begin{array}{l}\text { Width } \\
(\mu \mathrm{m})\end{array}$ & Depth $(\mu \mathrm{m})$ \\
\hline Mix 1 & 110000 & 60 & 11.5 \\
Mix 2 & 110000 & 60 & 11.5 \\
3 & 500 & 60 & 11.5 \\
4 & $(3500$-Bead Bed $)$ & 105 & 11.5 \\
Bead Bed & Varies & 105 & 11.5 \\
Weir & 150 & 85 & 4.5 \\
7 & 815 & 105 & 11.5 \\
Dilution & 110000 & 64 & 11.5 \\
Waste & 18330 & 105 & 11.5 \\
\hline
\end{tabular}

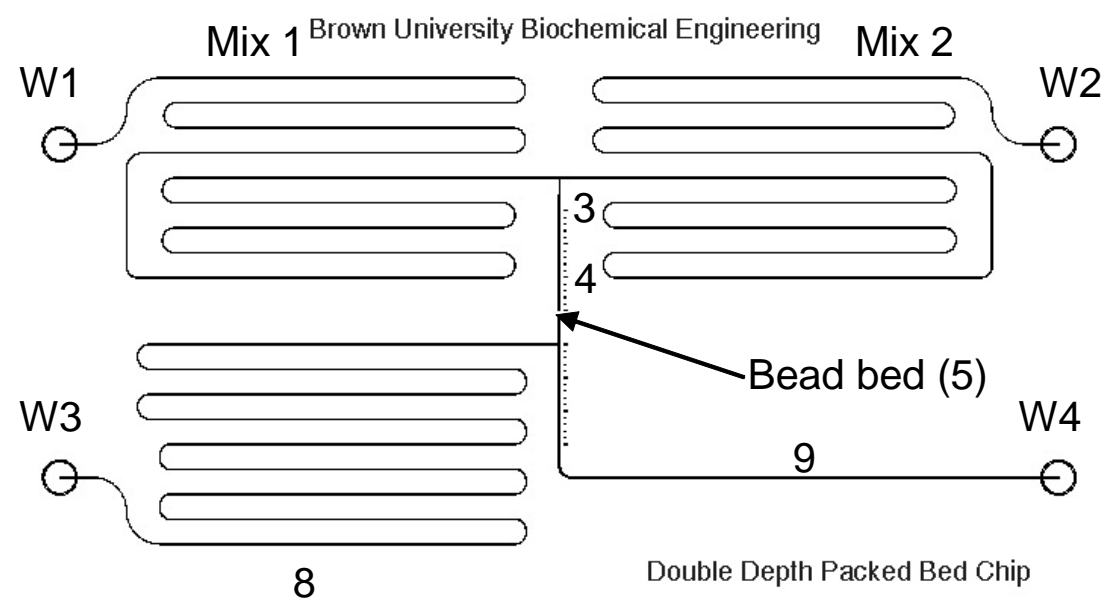

Figure 1: This mask defines the geometry of the microfluidic reactor chip. The reagent reservoirs W1-W4 are connected by a network of microchannels. 


\section{Flow control:}

Flow in each channel was regulated independently by controlling the air pressure at each well using peristaltic pumps and pressure sensors in a feedback arrangement controlled through a custom LabView interface (National Instruments). Each pressure port has accuracy to within $0.01 \mathrm{psi}$ of the assigned value over a $15 \pm 0.01 \mathrm{psi}$ range and response time of $5 \mathrm{psi} / \mathrm{sec}$. The microfluidic chip dilutions and incubation times are controlled by solving a system of momentum and continuity equations to derive the appropriate pressure condition. Pressure control reduces the reagent requirement to 6uL/well and eliminates the holdup volume found with syringes and feed lines. To improve dilution control on the chip, the high flow resistance of the packed bed is compensated proportionally distributing the pressure drop between the high-resistance packed bed and paired dilution channels (figure 1).

\section{Solution phase experiments:}

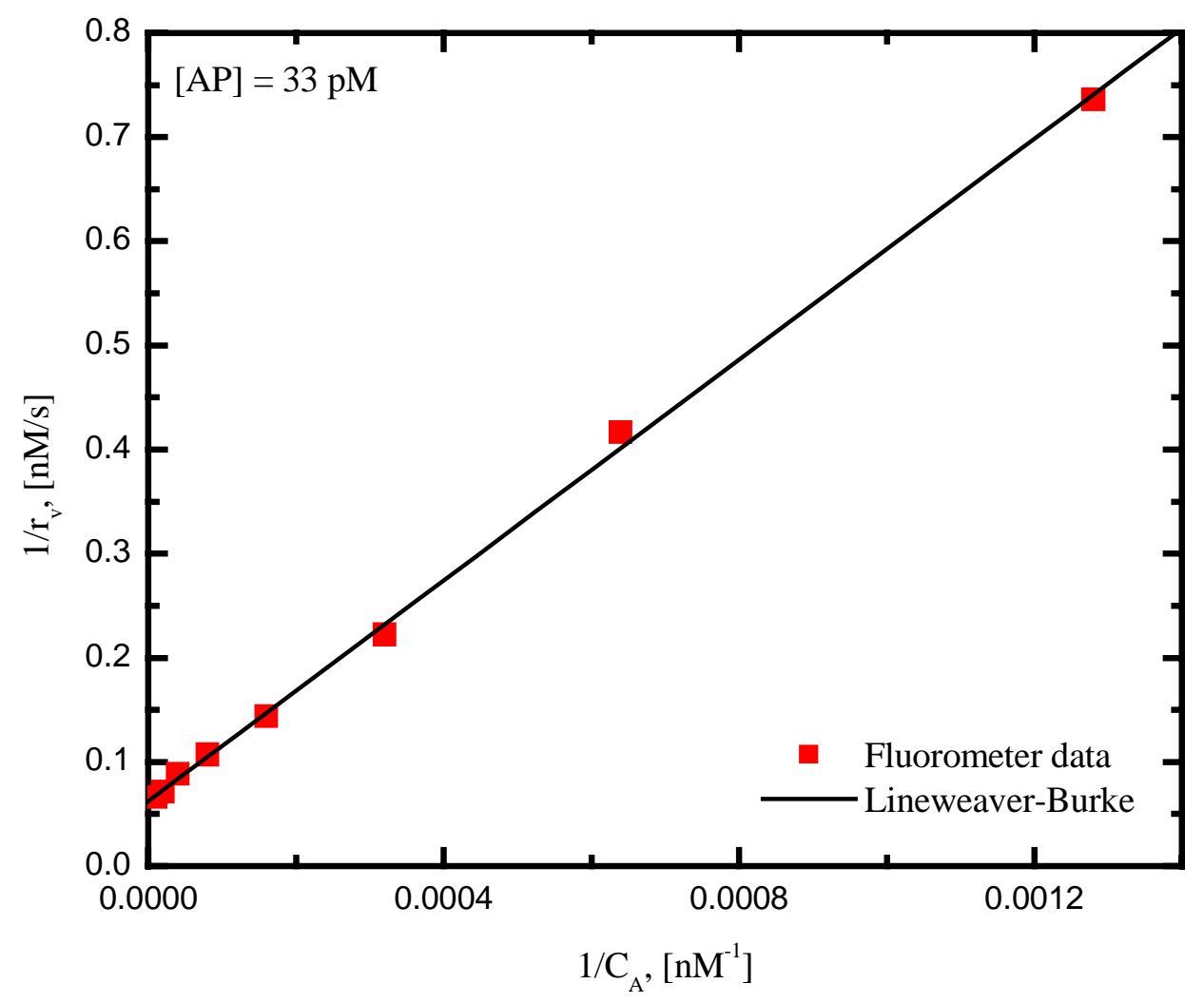

Figure 2: A Lineweaver-Burke kinetic plot for the solution phase alkaline phosphatase reaction.

\section{Packed bed flow control:}

To prepare for the packed bed kinetics test, it was first necessary to experimentally find the pressures to produce correct dilutions of reagents from the wells. A 50\% dye-buffer dilution was created by applying 3 psi to buffer well 1 and dye well 2 while holding waste well 4 at 0psi and blocking well 3 to create zero flow. Pressures on wells 1 and 2 were balanced to maintain a constant flow rate, while the side arm contribution at the tee junction varied from $0-100 \%$. A pressure of $1 \mathrm{psi}$ on well 1 and $5 \mathrm{psi}$ on well 2 contributed $100 \%$ DiFMU. Once verified experimentally, a pressure script was written to dilute DiFMU from $100 \%$ to $0 \%$ in $10 \%$ steps (figure 3a). Excess pressure, creating backflow in other arms of the chip, 
was applied briefly before and after the 10 step increments to ensure the accuracy of the $100 \%$ and $0 \%$ fluorescence intensity readings. The PMT detector was positioned across the channel immediately following the shallow weir where data was collected at 10Hz. The PMT measured the total fluorescence across the channel after the bead bed shown in figure $3 \mathrm{~b}$.

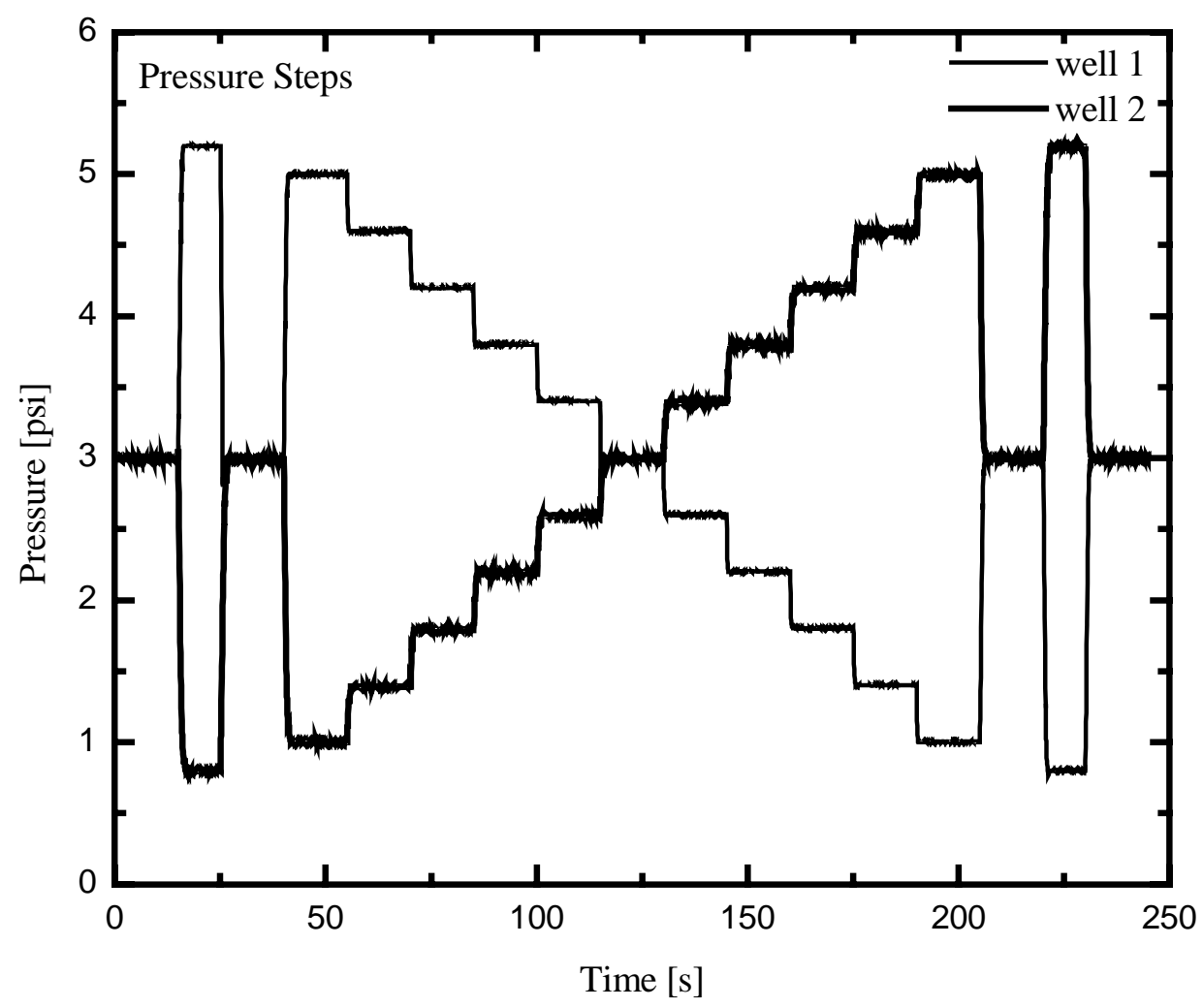

Figure 3a: The measured pressure profile for diluting substrate in the chip from 0 to $100 \%$. 


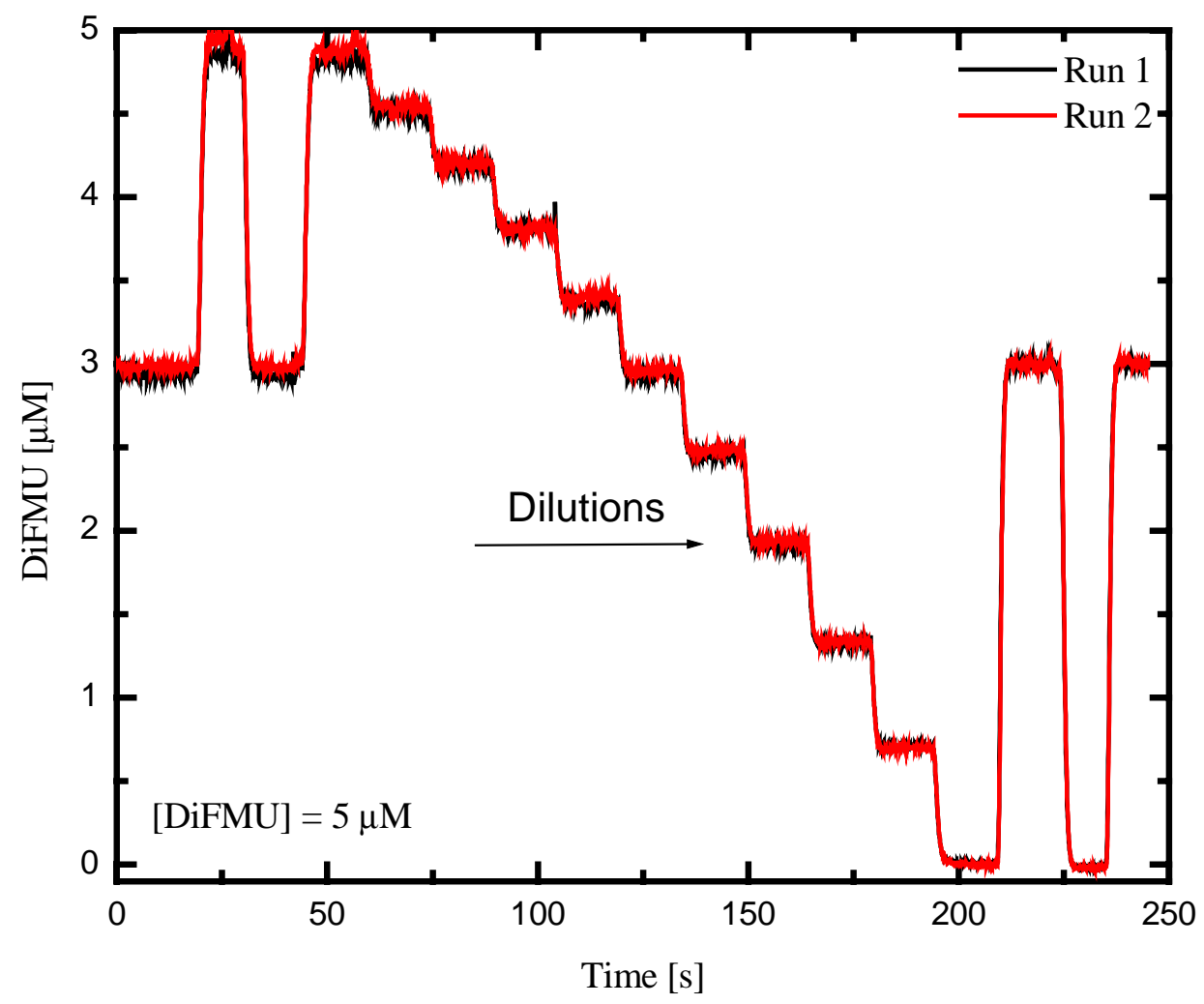

Figure 3b: The measured fluorescence intensity for $5 \mathrm{uM} \mathrm{DiFMU}$ dilution at $\mathrm{T}=23^{\circ} \mathrm{C}$.

1. Madou, M.J., Fundamentals of Microfabrication: The Science of Minaturization. 2002, Irvine: CRC Press. 